\title{
BRCA1 germline mutation and glioblastoma development: report of cases
}

\author{
Meriem Boukerroucha ${ }^{1}$, Claire Josse ${ }^{1,2^{*}}$, Karin Segers ${ }^{3}$, Sonia El-Guendi ${ }^{1}$, Pierre Frères ${ }^{2}$, Guy Jerusalem² \\ and Vincent Bours ${ }^{1,3}$
}

\begin{abstract}
Background: Germline mutations in breast cancer susceptibility gene 1 (BRCA1) increase the risk of breast and ovarian cancers. However, no association between BRCA1 germline mutation and glioblastoma malignancy has ever been highlighted.

Here we report two cases of BRCA1 mutated patients who developed a glioblastoma multiform (GBM).

Cases presentation: Two patients diagnosed with triple negative breast cancer (TNBC) were screened for BRCA1 germline mutation. They both carried a pathogenic mutation introducing a premature STOP codon in the exon 11 of the BRCA1 gene. Few years later, both patients developed a glioblastoma and a second breast cancer. In an attempt to clarify the role played by a mutated BRCA1 allele in the GBM development, we investigated the BRCA1 mRNA and protein expression in breast and glioblastoma tumours for both patients. The promoter methylation status of this gene was also tested by methylation specific PCR as BRCA1 expression is also known to be lost by this mechanism in some sporadic breast cancers.

Conclusion: Our data show that BRCA1 expression is maintained in glioblastoma at the protein and the mRNA levels, suggesting that loss of heterozygosity $(\mathrm{LOH})$ did not occur in these cases. The protein expression is tenfold higher in the glioblastoma of patient 1 than in her first breast carcinoma, and twice higher in patient 2. In agreement with the high protein expression level in the GBM, BRCA1 promoter methylation was not observed in these tumours.

In these two cases, despite of a BRCA1 pathogenic germline mutation, the tumour-suppressor protein expression is maintained in GBM, suggesting that the BRCA1 mutation is not instrumental for the GBM development.
\end{abstract}

Keywords: $B R C A 1$, Glioblastoma, Breast cancer

\section{Background}

Breast cancer susceptibility gene 1 (BRCA1) is the first tumour suppressor gene identified in familial breast cancer. Located on chromosome 17 (17q21), this gene encodes a multifunctional protein involved in several cellular processes such as DNA repair, chromatin remodelling and cell cycle regulation [1]. Several studies reported that germline mutations in $B R C A 1$ gene increase the risk to develop breast and ovarian cancers $[1,2]$. Indeed, women bearing pathogenic germline

\footnotetext{
* Correspondence: c.josse@ulg.ac.be

'University of Liège, GIGA-Cancer Research, Human Genetics Unit, Liège, Belgium

${ }^{2}$ Division of Medical Oncology, Liège University and CHU Sart Tilman Liège, Liège, Belgium

Full list of author information is available at the end of the article
}

BRCA1 mutations have a $45 \%$ to $80 \%$ risk to develop breast cancer by age 70 , and $36 \%$ to $66 \%$ for ovarian cancer [3]. BRCA1 somatic mutations are very rare but its promoter methylation is reported to occur in about $7 \%$ to $30 \%$ of breast and ovarian sporadic cancers $[4,5]$. In glioma, genome-wide association studies have identified common genetic variations in 7 genes that increase glioma risk (TERT, EGFR, CCDC26, CDKN2A, CDKN2B, PHLDB1 and RTEL1) but BRCA1 is not among them and there is no known association between BRCA1 gene and glioblastoma multiforme (GBM) [6-8]. Indeed, Elmariah and co-authors reported the case of a patient mutated for BRCA1 who developed glioblastoma but they did not investigate BRCA1 mRNA and protein expression in the GBM [9]. Piccirilli and co-authors have also studied 11 cases of GBM 
occurring after mammary carcinoma, but their genomic status concerning BRCA1 was unknown [10].

Here, we report two cases of patients with $B R C A 1$ germline mutation treated for breast cancer who developed glioblastoma few years after breast cancer diagnosis. The first patient had a triple negative breast cancer (TNBC) and six years later, a glioblastoma multiforme. In a very similar pattern, the second patient developed also a triple negative breast cancer and five years later a glioblastoma. In an attempt to clarify the role played by a mutated $B R C A 1$ allele in the GBM development, we assessed BRCA1 mRNA and protein expression in the two tumour types for each patient. We also checked the BRCA1 promoter methylation status.

Ethical approval was obtained from the local institutional ethical board (Comité d'éthique hospitalo-facultaire universitaire de Liège) in compliance with the Helsinki declaration, with the approval file number $n^{\circ} 2010 / 229$.

\section{Cases presentation}

\section{Patient 1}

A 28-years-old woman was diagnosed in 2000 with a ductal carcinoma of the left breast.

After radical mastectomy, histologic analysis revealed a $20 \mathrm{~mm}$ tumour with an infiltrating ductal carcinoma. Immunologic analysis demonstrated no expression of oestrogen and progesterone receptors (ER- and PR-) and no overexpression of HER2.

The patient was staged as T1N0M0 stage IA and received FEC adjuvant chemotherapy (FEC: Fluorouracil, Epirubicin, Cyclophosphamide). The proliferation marker Ki67 was expressed by $50 \%$ of the tumour cells.

Six years later, the patient developed a glioblastoma. After complete macroscopic surgical resection, the tumour was characterized as stage IV according to the WHO classification. The proliferative marker Ki67 was expressed by $40 \%$ of the tumour cells. The patient received temozolomide chemotherapy and radiotherapy followed by chemotherapy alone (6 cycles).

Two years after her diagnosis of GBM, she developed a carcinoma in the right breast. After mastectomy, the histologic and immunologic analysis of the $26 \mathrm{~mm}$ tumour revealed an infiltrating ductal carcinoma, negative for oestrogen and progesterone receptors but with HER2 gene amplification. The patient was staged as T2NOM0 stage IIA and received adjuvant chemotherapy targeted therapy (docetaxel and trastuzumab) for one year.

She died in 2012 after two relapses of the GBM.

$B R C A 1$ genetic testing was performed after the first breast cancer. The family tree is represented in Figure 1.A.

\section{Patient 2}

A 56-years-old woman was diagnosed in 2005 with a ductal carcinoma in the left breast. After mastectomy, histological analysis revealed a $20 \mathrm{~mm}$ tumour with an infiltrating ductal carcinoma. Immunologic analysis demonstrated absence of hormone receptors expression (ER-, PR-) as well as absence of HER2 overexpression. Lymph nodes were not infiltrated. The tumour was classified as T2NOMx stage IIA. The patient was treated with 6 cures of FEC chemotherapy. She developed metastases at the level of cervical and dorsal vertebra and received palliative chemotherapy (paclitaxel) and zoledronic acid.

Considering the family history of the patient (Figure 1B) and after molecular analysis of BRCA1 gene, the patient was subjected to oophorectomy and hysterectomy.

Five years later, the patient developed a second breast carcinoma in the right breast. After mastectomy, histologic analysis revealed an $40 \mathrm{~mm}$ in situ ductal carcinoma associated with a $2 \mathrm{~mm}$ infiltrating ductal carcinoma and the absence of sentinel lymph node infiltration. Immunologic analysis of the invasive carcinoma demonstrated the absence of hormone receptors expression and an absence of HER2 protein overexpression. The invasive tumour was classified as pT1aNOMx with a proliferative index based on Ki67 expression of 35\%.

Few days after tumour resection, the patient presented mental confusion and a brain scan showed a mass at the fronto-insular level. After surgical excision, the histologic analysis revealed a glioblastoma characterized as stage IV according to the WHO classification. The patient received temozolomide chemotherapy and radiotherapy. One year later, the patient had surgical resection of progressive glioblastoma.

She died in 2011.

\section{Molecular analysis \\ DNA isolation}

Tumour determined by a pathologist was manually macro-dissected from FFPE tissues. DNA was isolated from the first triple negative breast carcinoma and from the GBM in the two patients.

Blood samples were also collected in both patients to establish the BRCA1 genomic status, after genetic counselling. Genomic DNA from leucocytes was extracted by standard phenol procedure.

\section{BRCA1 gene analysis}

All coding exons of $B R C A 1$ gene were subjected to PCR amplification. Amplicons were denatured, heteroduplexed and evaluated for the presence of mutations by Denaturing High Performance Liquid Chromatography (DHPLC) using product-specific melting and solvent conditions. All amplicons showing abnormal DHPLC pattern were sequenced by Sanger sequencing using ABI 3130 and following manufacturer recommendations. 


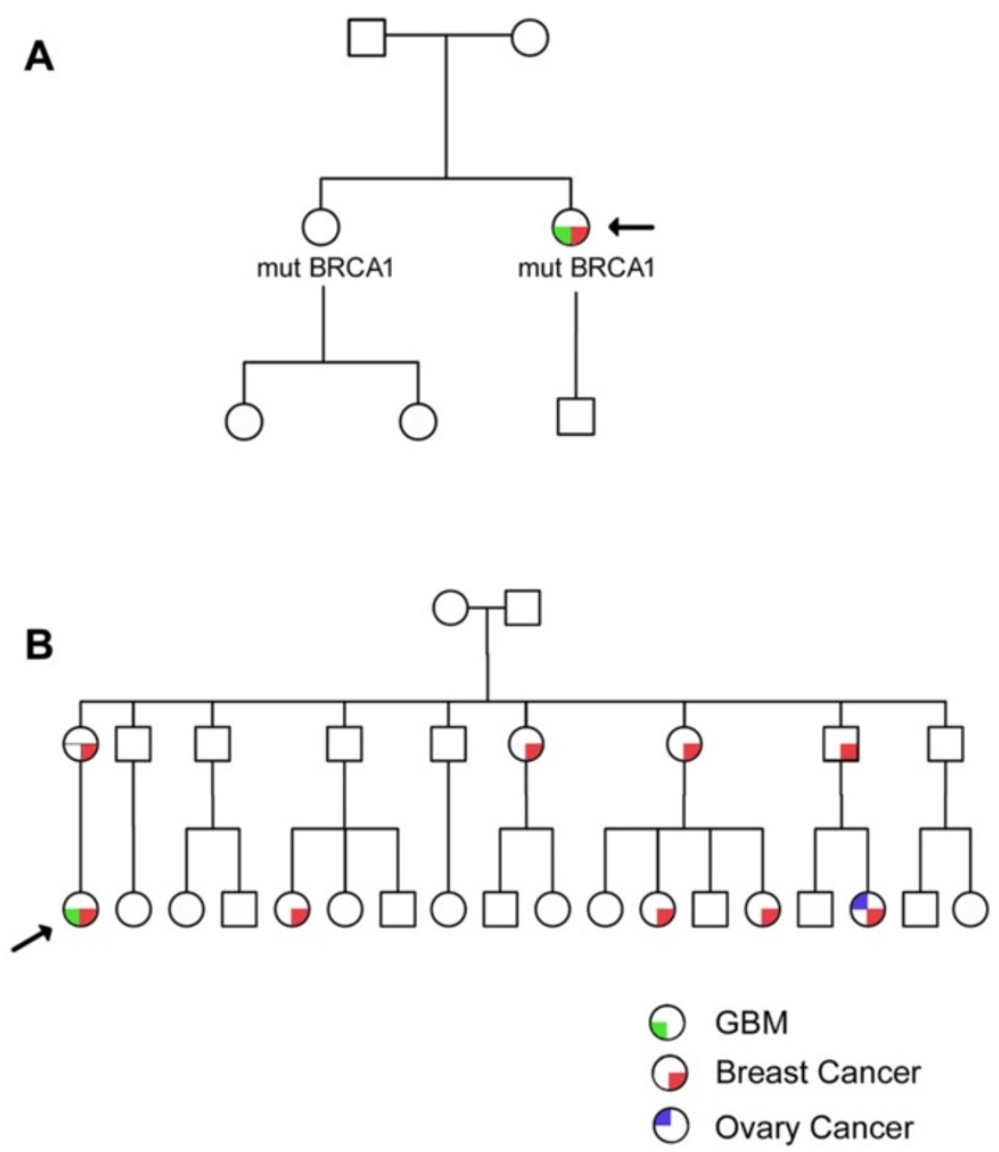

Figure 1 Family trees of the patients. A. Patient 1(arrow) B. Patient 2 (arrow). Cancer affected individuals are indicated.

The reference sequence BRCA1 (NM_007294.3) has been used for HGVS-approved amino acid numbering.

The following pathogenic mutations were founded by this method:

Patient 1: c.3481_3491del (p.Glu1161Phefs*3). This deletion of $11 \mathrm{bp}$ leads to a frame-shift and premature Stop codon. This mutation has been previously identified in our population.

Patient 2: c.2722G > T (p.Glu908*). This mutation induces a premature Stop codon and is frequently identified in our Belgian population (11\% of our BRCA1 mutations).

These mutations were confirmed on independent samples by direct Sanger sequencing.

Moreover, the screening for large genomic rearrangement was performed using Multiplex ligation dependent probe amplification MLPA kits P002-C2 (MRC Holland) on the BRCA1 gene region. This experiment did not highlight any abnormal genomic copy number change.

In attempt to identify $\mathrm{LOH}$, screening for the BRCA1 mutation has been performed by Sanger sequencing on DNA extracted from cerebral tumours of the two patients. However, the DNA quality obtained from GBM of patient 1 did not allow the amplification of the target region and the subsequent $B R C A 1$ sequencing. Regarding patient 2, the BRCA1 mutation was identified in heterozygous status, suggesting that no loss of heterozygosity occurred.

\section{$B R C A 1$ and $O^{6}$-methylguanine-DNA methyltransferase (MGMT) methylation status}

The methylation status of BRCA1 and MGMT promoter was assessed by methylation specific polymerase chain reaction (MSP-PCR) as previously described $[11,12]$.

DNA extracted from tumours was first treated by sodium bisulfite using the EZ DNA Methylation Kit (Zymo Research) following manufacturer recommendations. MSP-PCR was done following the protocol of Esteller and co-authors [13].

We assessed the methylation status of $B R C A 1$ promoter in the TNBC and GBM tumours for the two patients. A methylation was observed in TNBC tumour but not in GBM tumour for patient 1 as shown in Figure 2A. Regarding patient 2 , the $B R C A 1$ promoter was unmethylated in both tumours (Figure 2B).

On the other hand, the $M G M T$ promoter was methylated in GBM of both patients (Data not shown). 

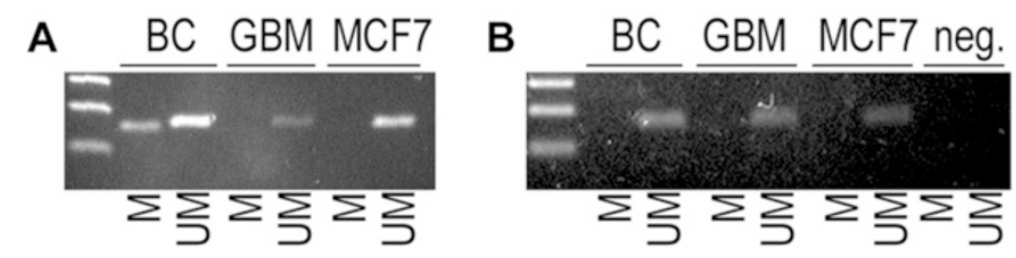

Figure 2 Methylation status of $B R C A 1$ promoter in tumours. A Patient 1. B Patient 2. $M=$ methylated, $U M=$ unmethylated, BC= breast cancer tissue, GBM = glioblastoma tissue, MCF7 = MCF-7 breast cancer cell line known to have unmethylated BRCA1 promoter.

\section{BRCA1 mRNA expression}

The mRNA expression was assessed by in situ hybridization using RNA scope technology (ACD) for FFPE samples. In this experiment, the target probes are designed as double- $Z$ as described by Fay Wang and co-authors [14]. The BRCA1 probe is complementary to the coding region aa369-1482, spanning exon 5 to exon 11 . To quantify the expression, spots and cells were independently and blindly counted twice, in subzones of the tumour. The ratio between the number of spots and the number of cells was compared to those of a positive control (MCF-7 cell line).

The mRNA expression level was $17,7 \%$ in breast carcinoma and 8\% in GBM of patient 1.

Regarding patient 2, the mRNA expression level was $10,24 \%$ in breast tumour and $12,67 \%$ in GBM tumour. Representative images of the BRCA1 mRNA expression in breast tumour and in GBM of both patients are represented in Figure 3.

\section{BRCA1 protein expression}

The protein level expression was assessed by proximity ligation assay (Duolink in situ detection reagents Sigma). This assay is more specific than the conventional immunohistochemistry thanks to the use of two primary antibodies directed against two epitopes of the same protein. The primary antibodies are raised in different species and are recognized by two secondary antibodies coupled with oligonucleotide probes. After ligation of the two probes, the circular DNA is amplified by polymerase reaction. The detection is performed using horse radish peroxidase (HRP) labelled probes and a chromogenic reaction using 3,3'Diaminobenzidine (DAB). In order to generate a signal only with the full length $B R C A 1$ protein, the two primary antibodies where chosen to be specific to the N-term ([MS110] ab16780, Abcam) and C-term domain (Sigma, SAB4502848) of the $B R C A 1$ protein, respectively.

The protein expression level was estimated using the same quantification method as for mRNA expression level, as compared to MCF7 cells.

The protein expression level was $4,08 \%$ in breast tumour and $45,86 \%$ in GBM of patient 1 and $19,04 \%$ in breast tumour and 36,37\% in GBM of patient 2. Representative images of the BRCA1 protein expression in GBM of both patients are represented in Figure 4.

Promoter methylation, mRNA expression and protein expression levels of BRCA1 are summarized in Table 1.

\section{Discussion}

Here, we report the cases of two patients, with a germline BRCA1 mutation, who developed two primary breast cancers and a GBM. Both women present a germline pathogenic heterozygote mutation in the exon 11 of the BRCA1 gene, leading to a truncated transcript.

In $80 \%$ of $B R C A 1$ breast carcinoma the protein expression is lost because of the deletion of the second allele $[15,16]$. Few cases of glioblastoma in BRCA1 mutation carriers were reported but the $B R C A 1$ expression status has never been studied.

In an attempt to clarify the role played by a $B R C A 1$ mutation in GBM development, we performed diverse molecular experiments to characterize the expression status of BRCA1 in glioblastoma, and in the first TNBC of the two patients.

Our data show that the BRCA1 protein expression is maintained in glioblastoma suggesting that no loss of heterozygosity occurred in these tumours. The sequencing data of the tumoural BRCA1 gene in the GBM of patient 2 support this hypothesis. The protein expression is tenfold higher in the glioblastoma of patient 1 than in her breast carcinoma, and twice higher in patient 2 . However, the BRCA1 expression level was never completely lost, even in the TNBC. This observation is in concordance with what is observed in breast cancer cell lines where BRCA1 is mutated (HCC1937) or its promoter methylated (UACC3199) $[17,18]$.

$B R C A 1$ expression is also known to be lost in some sporadic breast cancers after methylation of the gene promoter (13\% of cases) [12,19], but promoter methylation is rarely observed in tumours of $B R C A 1$ mutation carriers [20]. However, we observed BRCA1 promoter methylation in the triple negative breast carcinoma of patient 1, but not in glioblastoma. In agreement with the high protein expression level in the GBM, patient 2 did not present any $B R C A 1$ promoter methylation in this 


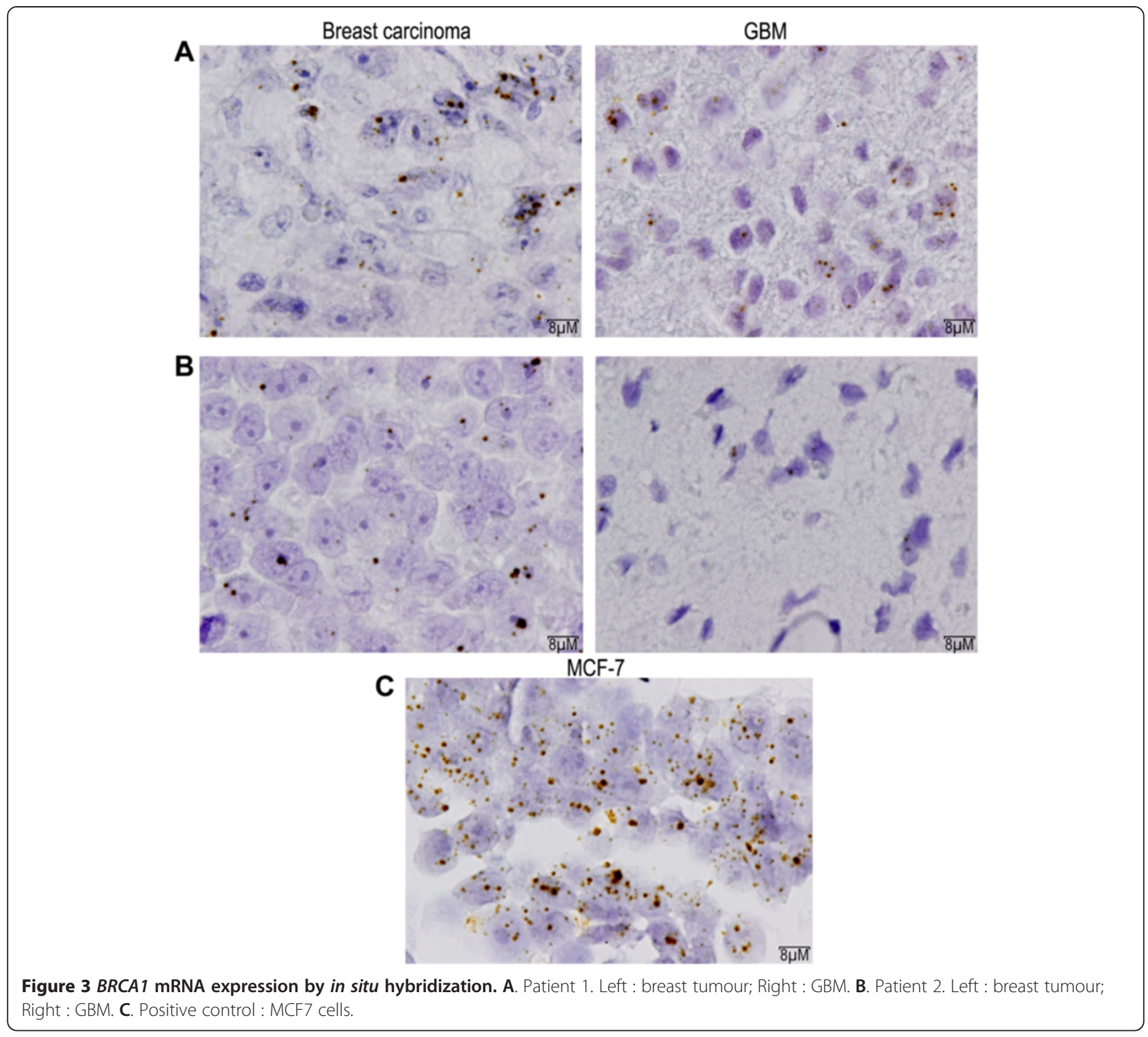

tumour. Therefore, in these two cases, despite of a $B R C A 1$ pathogenic germline mutation, the tumoursuppressor protein expression is maintained in GBM, suggesting that the BRCA1 mutation is not instrumental for GBM development. This observation is consistent with cancer statistics that have not highlighted any increased risk for brain tumour development in BRCA1 carriers [21,22]. However, these studies were conducted in 1994 and 2002. Since then, treatment of breast cancer has evolved and survival increased, maybe allowing the detection of a previously hidden link between mutation of $B R C A 1$ and the risk to develop GBM. Indeed, as breast cancer appears before GBM in BRCA1 mutated patients, increased survival because of improved treatments may now allow the recording of brain tumour development. Moreover, the work of Konishi and coauthors has demonstrated that a heterozygous mutation of $B R C A 1$ without loss of the wild type allele can still induce genome instability [23]. Thus, conserved BRCA1 protein expression in glioblastoma does not completely rule out its role in GBM development.

Another way to establish a relation between the BRCA1 mutation and the GBM development would be to estimate the difference between the observed incidence of cases and the theoretical occurrence risk of both events. In our European population and during the last 10 years of BRCA1 mutation screening, approximately between 2 and 8 cases associating BRCA1 mutation and GBM for 100 million women are expected [24-26], but only three cases have been reported in the literature (two in Europe in the same 


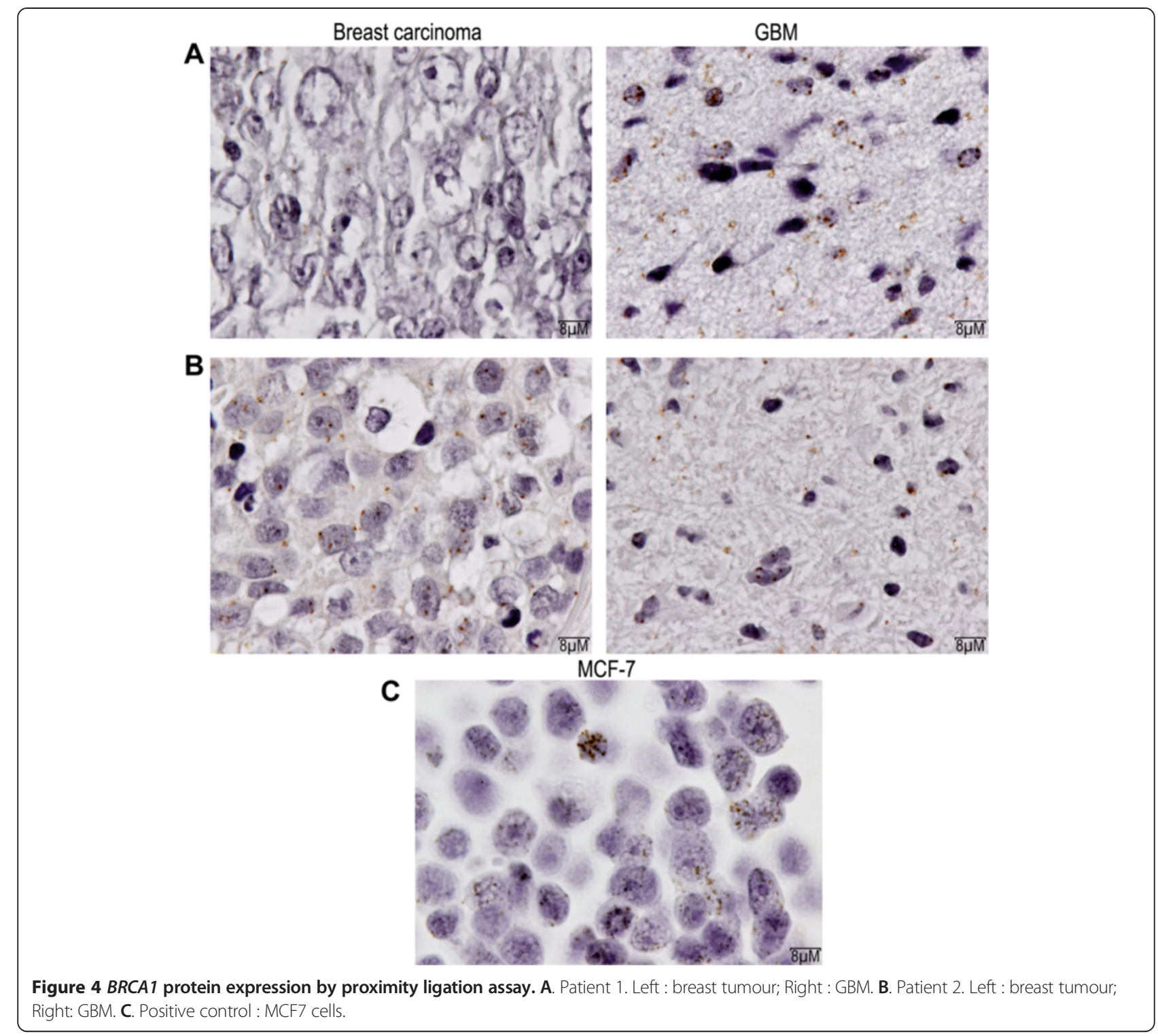

centre and one in United States). Moreover, we are not aware of any case of GBM in males with BRCA1 mutation.

\section{Conclusion}

Our study failed to establish any biological link between GBM and BRCA1 mutation but further genotype/phenotype studies might be needed to finally demonstrate or exclude any relation. It is possible to imagine that phenotype specificities could be linked with an increased risk of unusual cancers in BRCA1 mutated patients. Indeed our two patients had mutations generating truncated proteins. Moreover, they both developed bilateral breast cancers. Large studies are required to verify whether complete loss of function BRCA1 mutations or interactions with other genes could be specifically associated with increased risk of rare cancers.

Table 1 Summary of BRCA1 molecular analysis

\begin{tabular}{llllllll}
\hline & Breast carcinoma & & & Glioblastoma & \\
\cline { 2 - 4 } & BRCA1 promoter methylation & $\begin{array}{l}\text { BRCA1 mRNA } \\
\text { (\%MCF7) }\end{array}$ & $\begin{array}{l}\text { BRCA1 protein } \\
\text { (\%MCF7) }\end{array}$ & & BRCA1 promoter methylation & $\begin{array}{l}\text { BRCA1 mRNA } \\
\text { (\%MCF7) }\end{array}$ & $\begin{array}{l}\text { BRCA1 protein } \\
\text { (\%MCF7) }\end{array}$ \\
\hline Patient 1 & Yes & 18 & 4 & No & No & 8 & 46 \\
Patient 2 & No & 10 & 19 & & 13 & 36 \\
\hline
\end{tabular}




\section{Consent}

Written informed consent was obtained from one direct relative of each patient for publication of these case reports and accompanying images.

A copy of the written consent is available for review by the Editor of this journal.

\section{Competing interests}

The authors declare that they have no competing interests.

\section{Authors' contributions}

MB carried out the molecular analysis, participated in the design of the study and drafted the manuscript. CJ participated in the design of the study and the writing of the manuscript. KS performed some molecular analysis and participated in the writing of the manuscript. SE performed some molecular analysis. PF collected clinical data. VB and GJ conceived of the study, and participated in its design and coordination and helped to draft the manuscript. All authors read and approved the final manuscript.

\section{Acknowledgments}

We first thank the patients and their family. We also thank the Biobanque of Liège University Hospital, the GIGA-immunohistology platform, the GIGABioinformatic platform, and the GIGA-Imaging platform. We thank Dr Joelle Collignon, Dr Hélène Schroeder, Bouchra Boujemla, Jerome Thiry, Corinne Fasquelle, Fabienne Perin, Tiberio Sticca and Stéphane Wenric. MB is funded by Télévie-FRS-FNRS. SE is funded by FRIA-FNRS. PF is funded by FRS-FNRS. This work was supported by the F.I.R.S.-CHU; Belgian Funds for Scientific Research (F.R.S.-FNRS); Centre anti-Cancéreux; PPP grant from Région Wallonne and F.R.S.-FNRS-Télévie.

\section{Author details}

'University of Liège, GIGA-Cancer Research, Human Genetics Unit, Liège, Belgium. ${ }^{2}$ Division of Medical Oncology, Liège University and CHU Sart Tilman Liège, Liège, Belgium. ${ }^{3}$ Human Genetics Department, Liège University Hospital, Liège, Belgium.

Received: 26 May 2014 Accepted: 17 March 2015

Published online: 26 March 2015

\section{References}

1. Hall JM, Lee MK, Newman B, Morrow JE, Anderson LA, Huey B, et al. Linkage of early-onset familial breast cancer to chromosome 17q21. Science. 1990:250:1684-9.

2. Miki Y, Swensen J, Shattuck-Eidens D, Futreal PA, Harshman K, Tavtigian S, et al. A strong candidate for the breast and ovarian cancer susceptibility gene BRCA1. Science. 1994;266:66-71.

3. Satagopan JM, Offit K, Foulkes W, Robson ME, Wacholder S, Eng CM, et al. The lifetime risks of breast cancer in Ashkenazi Jewish carriers of BRCA1 and BRCA2 mutations. Cancer Epidemiol Biomarkers Prev. 2001;10:467-73.

4. Catteau A, Morris JR. BRCA1 methylation: a significant role in tumour development? Semin Cancer Biol. 2002;12:359-71.

5. Wei M, Grushko TA, Dignam J, Hagos F, Nanda R, Sveen L, et al. BRCA1 promoter methylation in sporadic breast cancer is associated with reduced BRCA1 copy number and chromosome 17 aneusomy. Cancer Res. 2005;65:10692-9.

6. Wrensch M, Jenkins RB, Chang JS, Yeh R-F, Xiao Y, Decker PA, et al. Variants in the CDKN2B and RTEL1 regions are associated with high-grade glioma susceptibility. Nat Genet. 2009:41:905-8.

7. Sanson M, Hosking FJ, Shete S, Zelenika D, Dobbins SE, Ma Y, et al. Chromosome 7p11.2 (EGFR) variation influences glioma risk. Hum Mol Genet. 2011;20:2897-904.

8. Shete S, Hosking FJ, Robertson LB, Dobbins SE, Sanson M, Malmer B, et al. Genome-wide association study identifies five susceptibility loci for glioma. Nat Genet. 2009;41:899-904.

9. Elmariah SB, Huse J, Mason B, Leroux P, Lustig RA. Multicentric glioblastoma multiforme in a patient with BRCA-1 invasive breast cancer. Breast J. 2006:12:470-4.

10. Piccirilli M, Salvati M, Bistazzoni S, Frati A, Brogna C, Giangaspero F, et al. Glioblastoma multiforme and breast cancer: report on 11 cases and clinico-pathological remarks. Tumori. 2005;91:256-60.
11. Rice JC, Massey-Brown KS, Futscher BW. Aberrant methylation of the BRCA1 CpG island promoter is associated with decreased BRCA1 mRNA in sporadic breast cancer cells. Oncogene. 1998;17:1807-12.

12. Esteller M, Silva JM, Dominguez G, Bonilla F, Matias-Guiu X, Lerma E, et al. Promoter hypermethylation and BRCA1 inactivation in sporadic breast and ovarian tumors. J Natl Cancer Inst. 2000;92:564-9.

13. Esteller M, Hamilton SR, Burger PC, Baylin SB, Herman JG. Inactivation of the DNA repair gene O6-methylguanine-DNA methyltransferase by promoter hypermethylation is a common event in primary human neoplasia. Cancer Res. 1999:59:793-7.

14. Wang F, Flanagan J, Su N, Wang L-C, Bui S, Nielson A, et al. RNAscope: a novel in situ RNA analysis platform for formalin-fixed, paraffin-embedded tissues. J Mol Diagn. 2012;14:22-9.

15. Cornelis RS, Neuhausen SL, Johansson O, Arason A, Kelsell D, Ponder BA, et al. High allele loss rates at 17q12-q21 in breast and ovarian tumors from BRCAl-linked families. The Breast Cancer Linkage Consortium. Genes Chromosomes Cancer. 1995:13:203-10.

16. Osorio A, de la Hoya M, Rodríguez-López R, Martínez-Ramírez A, Cazorla A, Granizo JJ, et al. Loss of heterozygosity analysis at the BRCA loci in tumor samples from patients with familial breast cancer. Int J Cancer. 2002;99:305-9.

17. Xu J, Huo D, Chen Y, Nwachukwu C, Collins C, Rowell J, et al. CpG island methylation affects accessibility of the proximal BRCA1 promoter to transcription factors. Breast Cancer Res Treat. 2010;120:593-601.

18. Tassone P, Tagliaferri P, Perricelli A, Blotta S, Quaresima B, Martelli ML, et al. BRCA1 expression modulates chemosensitivity of BRCA1-defective HCC1937 human breast cancer cells. Br J Cancer. 2003:88:1285-91.

19. Alvarez S, Diaz-Uriarte R, Osorio A, Barroso A, Melchor L, Paz MF, et al. A predictor based on the somatic genomic changes of the BRCA1/BRCA2 breast cancer tumors identifies the non-BRCA1/BRCA2 tumors with BRCA1 promoter hypermethylation. Clin Cancer Res. 2005;11:1146-53.

20. Lips EH, Mulder L, Oonk A, van der Kolk LE, Hogervorst FBL, Imholz ALT, et al. Triple-negative breast cancer: BRCAness and concordance of clinical features with BRCA1-mutation carriers. Br J Cancer. 2013;108:2172-7.

21. Ford D, Easton DF, Bishop DT, Narod SA, Goldgar DE. Risks of cancer in BRCA1-mutation carriers. Breast Cancer Linkage Consortium. Lancet. 1994;343:692-5.

22. Thompson D, Easton DF. Cancer Incidence in BRCA1 mutation carriers. J Natl Cancer Inst. 2002;94:1358-65.

23. Konishi H, Mohseni M, Tamaki A, Garay JP, Croessmann S, Karnan S, et al. Mutation of a single allele of the cancer susceptibility gene BRCA1 leads to genomic instability in human breast epithelial cells. Proc Natl Acad Sci U S A. 2011;108:17773-8.

24. Breast Cancer Statistics - Cancer Research UK. [http://www.cancerresearchuk. org/cancerinfo/cancerstats/types/breast/].

25. Fackenthal JD, Olopade OI. Breast cancer risk associated with BRCA1 and BRCA2 in diverse populations. Nat Rev Cancer. 2007;7:937-48.

26. Stupp R, Roila F. Malignant glioma: ESMO clinical recommendations for diagnosis, treatment and follow-up. Ann Oncol. 2009:20 Suppl 4:126-8.

\section{Submit your next manuscript to BioMed Central and take full advantage of:}

- Convenient online submission

- Thorough peer review

- No space constraints or color figure charges

- Immediate publication on acceptance

- Inclusion in PubMed, CAS, Scopus and Google Scholar

- Research which is freely available for redistribution 